\title{
SAND2000-0049C
}

\section{SINGLE-STEP ASSEMBLY OF COMPLEX 3-D MICROSTRUCTURES}

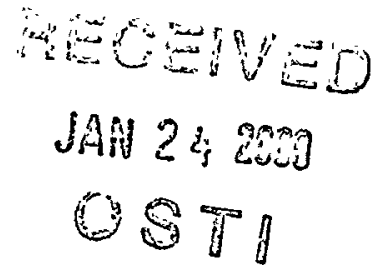

Elliot E. Hui, Roger T. Howe, and M. Steven Rodgers*

Berkeley Sensor \& Actuator Center, University of California, Berkeley, CA 94720-1774, USA

*Intelligent Micromachine Dept., Sandia Natl. Laboratories, Albuquerque, NM 87185-1080, USA

\begin{abstract}
This paper describes three-dimensional microstructures fabricated in a planar process and assembled in a single step. Multiple plates are constrained by hinges in such a way as to reduce the assembly process to a single degreeof-freedom of motion. Serial microassembly of these structures is simpler; moreover, selfassembly using hydrodynamic forces during release is much more feasible than with earlier, multiple degree-of-freedom hinged structures. A $250-\mu \mathrm{m}$ corner cube reflector, a 6-sided closed box, and a 3-D model of the Berkeley Campanile clock tower have been demonstrated in the 4-level polysilicon SUMMiT MEMS foundry.
\end{abstract}

\section{INTRODUCTION}

\section{Background}

Surface micromachining processes inherently produce two-dimensional thin-film structures. However, three-dimensional structures may be achieved by rotating plates out of plane on hinges [1]. This technique has been utilized widely to create a number of complex and useful microstructures.

Assembly usually involves manually flipping and locking various elements into place using standard electrical probing equipment. A significant drawback is that this assembly process is labor-intensive and slow, and yield is often low since microstructures can be easily broken during assembly. Therefore, a technique to increase the speed and yield of assembly would make high-volume manufacturing of these structures more attractive.

\section{Concept}

The problem of assembling three-dimensional structures from surface micromachined parts is very similar to the problem of creating the three-dimensional structures that spring out of children's pop-up books (Fig. 1) As a page is turned, a flat, planar structure assembles itself into the third dimension due to the way the various paper elements are hinged together and constrained. For decades, artists have been perfecting clever designs for use in pop-up books.

This concept has been successfully used to design and fabricate three-dimensional MEMS structures which are assembled using actuation of a single polysilicon plate. These are referred to as "pop-up structures" in this paper. Compared with previous three-dimensional flip-up designs, assembly is much easier. In addition, complex structures have been achieved that would have been difficult or impossible to realize using other methods.

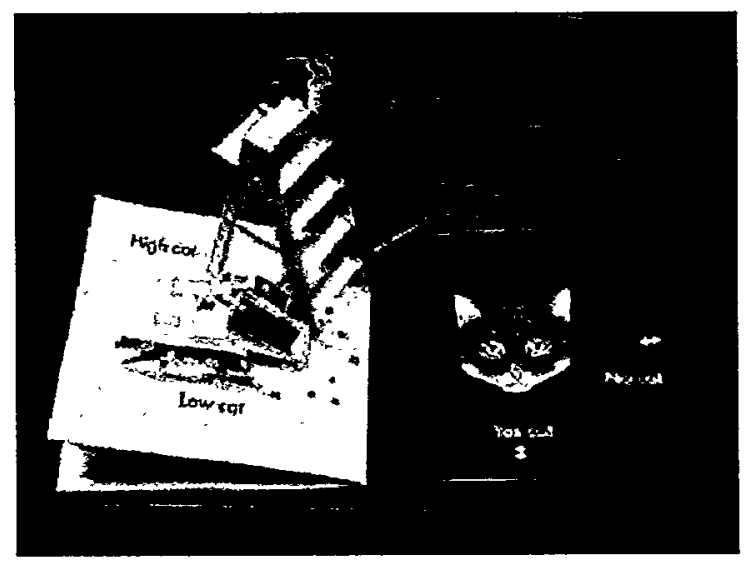

Figure 1: An example of a pop-up book 


\section{DISCLAIMER}

This report was prepared as an account of work sponsored by an agency of the United States Government. Neither the United States Government nor any agency thereof, nor any of their employees, make any warranty, express or implied, or assumes any legal liability or responsibility for the accuracy, completeness, or usefulness of any information, apparatus, product, or process disclosed, or represents that its use would not infringe privately owned rights. Reference herein to any specific commercial product, process, or service by trade name, trademark, manufacturer, or otherwise does not necessarily constitute or imply its endorsement, recommendation, or favoring by the United States Government or any agency thereof. The views and opinions of authors expressed herein do not necessarily state or reflect those of the United States Government or any agency thereof. 


\section{DISCLAIMER}

\section{Portions of this document may be illegible in electronic image products. Images are produced from the best available original document.}




\section{DESIGN}

\section{Single-step assembly}

An example of a conventional fold-up microstructure is the corner-cube-reflector (CCR) communications device [2] shown in Fig. 2. Excluding the actuated bottom plate, there are four plates to be flipped up and locked together for assembly, or four degrees-of-freedom.

In comparison, the pop-up CCR shown in Fig. 3 has five separate plates, all connected by hinges. The hinges introduce constraints that reduce the system to roughly a single degreeof-freedom. There is therefore a single path of motion that the system of plates can take from the planar unassembled state (Fig. 3a) to the assembled state (Fig. 3b), and the hinges constrain the system from straying from this path.

This motion can be initiated manually through the use of a micromanipulator. Flipping a single plate, the one analogous to the page of the pop-up book, accomplishes assembly of the entire system of plates. This plate may be designed in a fashion similar to an actual book page (Fig. 4); however this is not necessary.

Parallel self-assembly [5] of fold-up microstructures can be accomplished through the use of hydrodynamic forces to flip plates into position [1]. With regard to self-assembly, a single degree-of-freedom in assembly is attractive. Greater degrees of freedom lower the probability that all plates will simultaneously come together in just the right configuration. The tendency to self-assemble may be further enhanced by the addition of structural features to interact with the fluid flow.

\section{Hinges}

Conceptually, the hinges used in these structures are similar to the substrate hinge described in [1], with a pin rotating within a staple. The use of the Sandia SUMMiT [3] fabrication process, however, provides three

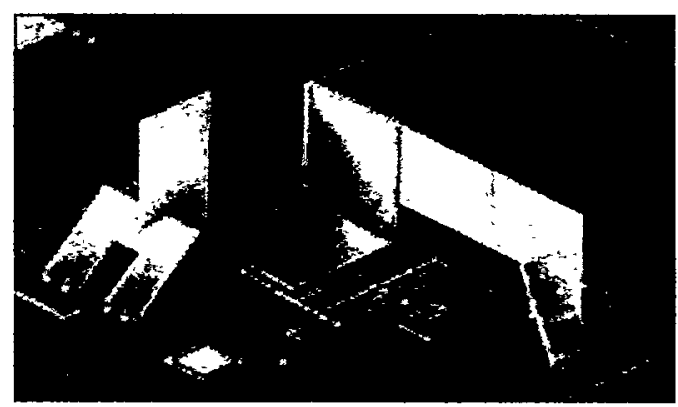

Figure 2: A corner-cube reflector with a typical flip-up and lock design.
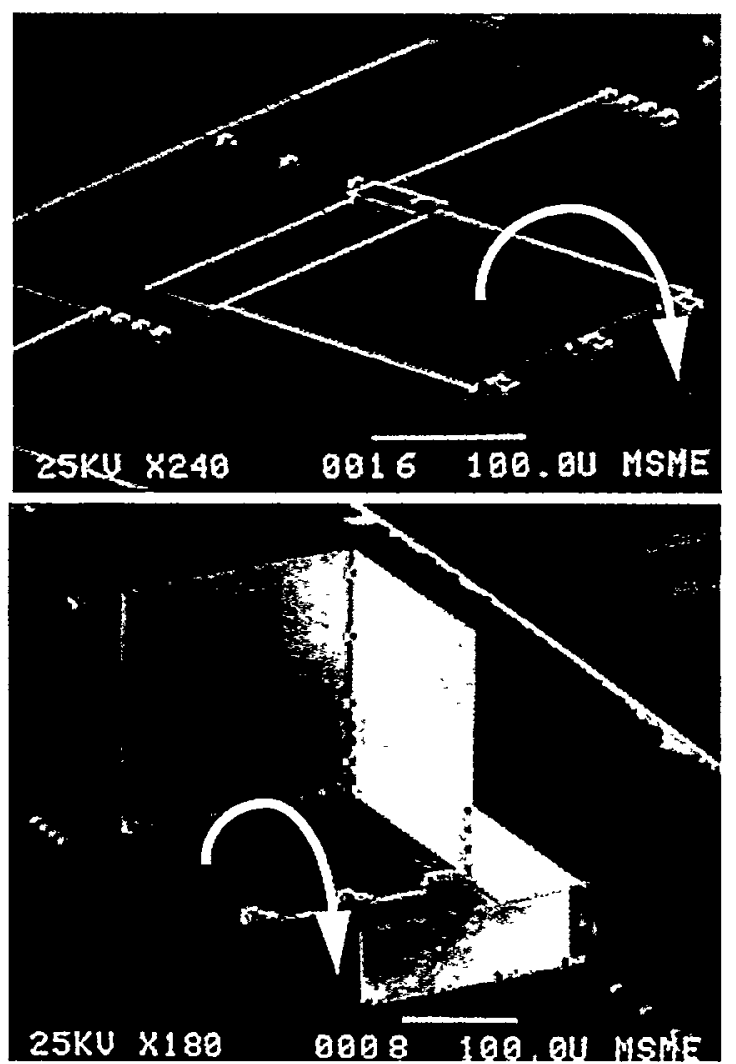

Figure 3: A CCR designed for single-step assembly: a) unassembled; b) assembled

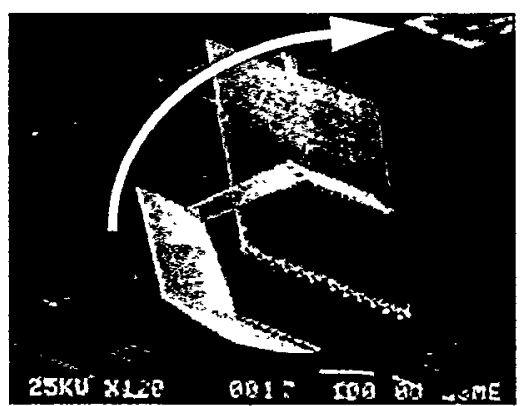

Figure 4: A similar CCR in mid-assembly 
mechanical layers of polysilicon, allowing for such pin-in-staple hinges to be used for plateto-plate connections (Fig. 5) as well as the typical plate-to-substrate connections. This type of plate-to-plate hinge provides a more precise connection than a scissor hinge [1] and exerts less stress on the structural elements than a torsion-bar hinge.

When used for a plate-to-substrate connection however, a standard substrate hinge design in the SUMMiT process results in a hinge pin that can move considerably within its constraining staple (Fig. 6). This amount of play can often allow the attached hinged plate to jam (Fig. 7), and thus poorly constrained hinges result in low yield. This poor level of constraint is due to the fact that the first structural polysilicon layer (Poly 1 ) is only $1-\mu \mathrm{m}$ thick, compared to a $2-\mu \mathrm{m}$ thick first sacrificial layer (Oxide1).

However, another aspect of the SUMMiT process can be utilized to create an alternative substrate hinge design that is highly precise (Fig. 8). As shown in Figure 9, following the deposition of the first structural polysilicon layer, selected areas of the previous sacrificial layer can be removed. Properly utilized, this process step allows the hinge pin to be surrounded on top and bottom by the second polysilicon layer, creating a very tightly constrained hinge.

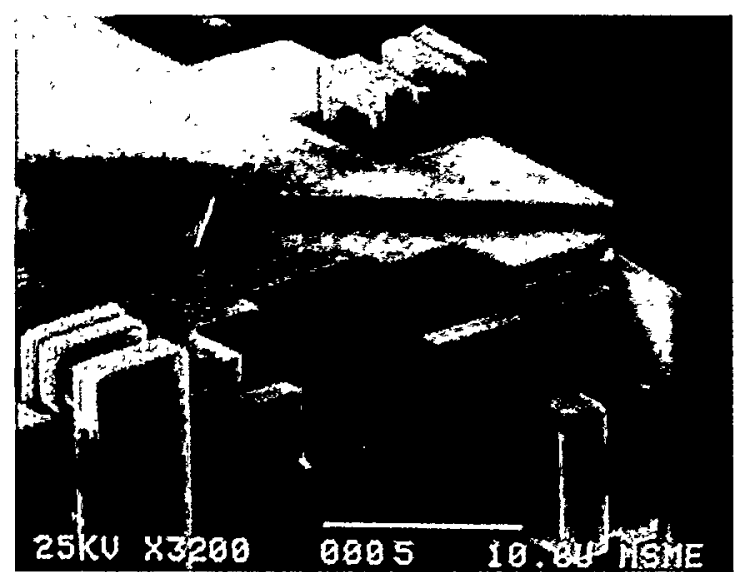

Figure 5: Three levels of structural polysilicon in the Sandia SUMMiT process allow for suspended pin-in-staple hinges, resulting in precise plate-to-plate connections.

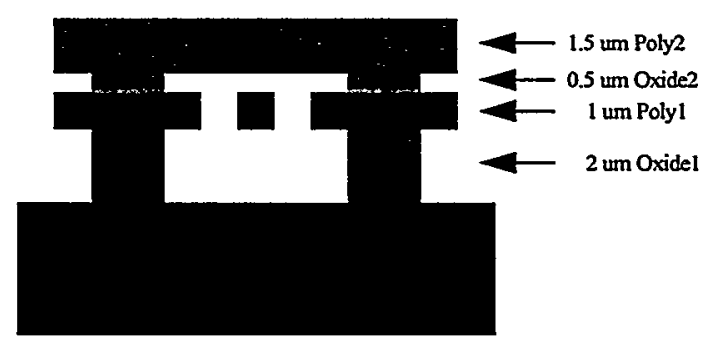

Figure 6: Cross-sectional view of a conventionally-designed substrate hinge in the SUMMiT process. The thin first structural layer makes this hinge poorly constrained.

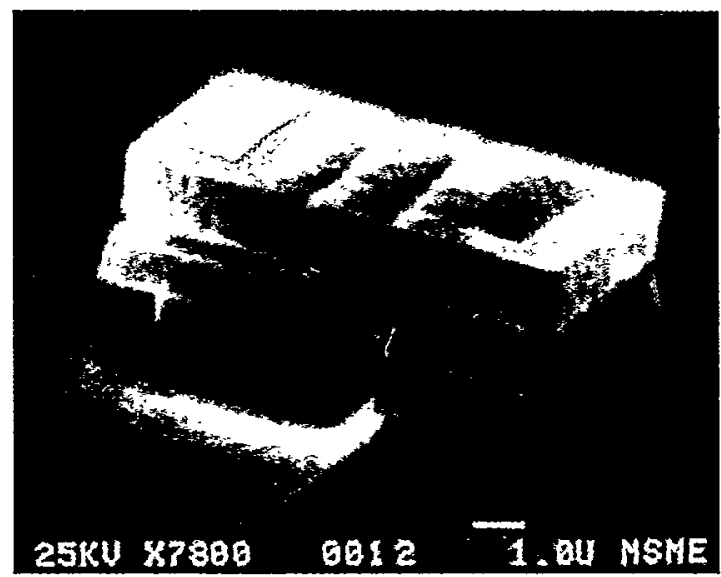

Figure 7: Conventional substrate hinge as diagramed in Fig. 6. The Poly 1 plate has slid into a position where it cannot rotate out of plane for assembly.

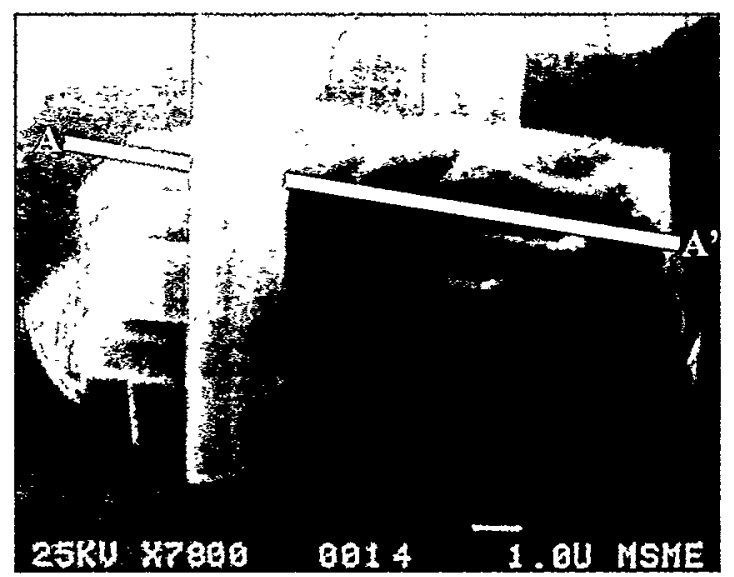

Figure 8: Alternative substrate hinge takes advantage of a SUMMiT-specific step to tightly constrain hinge pin. Here the hinged plate has been rotated into the assembled position. Fig. 9 illustrates cross-section A-A'. 


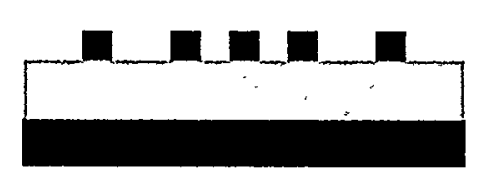

(a)

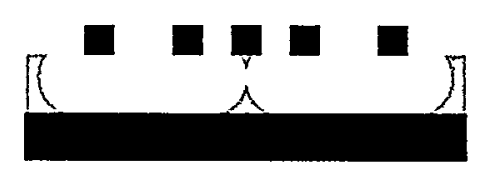

(b)

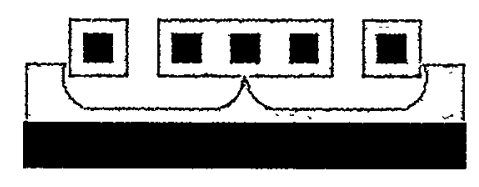

(c)

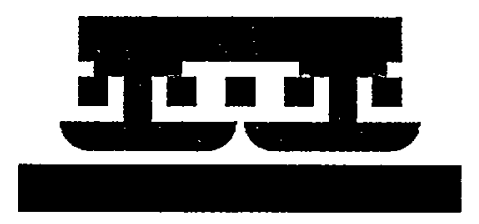

(d)

Figure 9: Simplified process flow for alternative substrate hinge. Illustrations are of cross-section A-A' from Figure 8. After deposition and patterning of Oxidel and Polyl (a), selected areas of Oxidel are etched out from under Polyl (b). Next, Oxide2 is deposited (c), and after patterning, Poly 2 is deposited (d). Poly 2 surrounds the hinge pin on top and bottom.

\section{Pop-up structure design}

Although a detailed analysis of the design methodology for pop-up structures is beyond the scope of this paper, one basic building block will be examined. Fig. 10 shows a schematic layout for a simple standing wedge. The assembled structure is shown above the layout. In this case, the Poly 3 plate is analogous to a book page, and the structure is assembled as this plate is turned. Note the placement of the hinges, which connect the plates along specific edges. In particular, note that the Polyl-tosubstrate and Poly2-to-Poly3 connections lie on overlapping edges.

If $\phi$ is $90^{\circ}$, the two standing plates will be orthogonal to the substrate when assembled, and the angle between the plates will be $2 \theta$. In
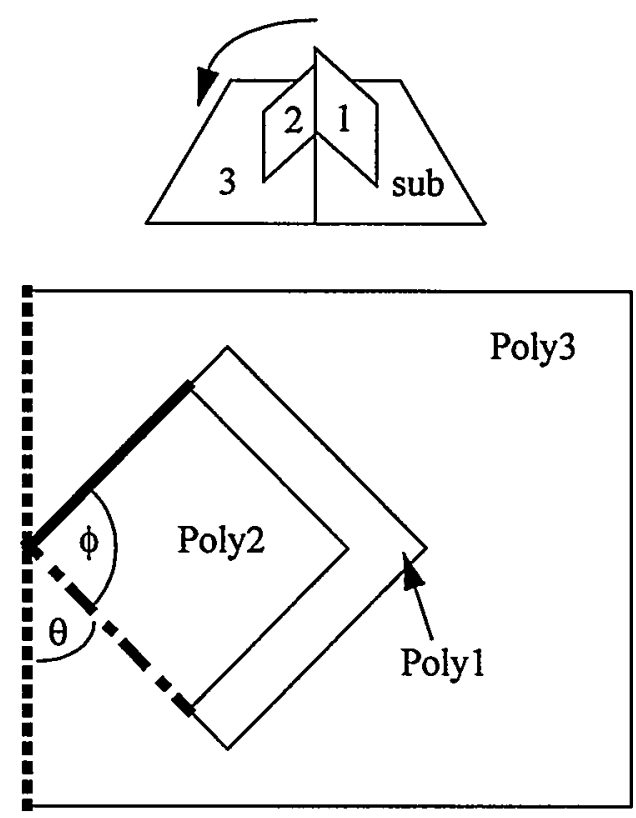

Location of hinge connections:

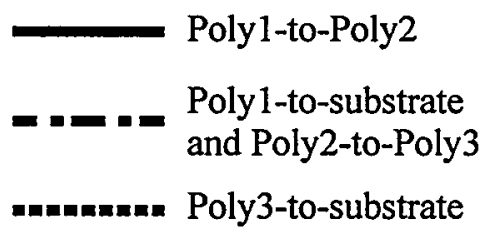

Figure 10: Schematic layout of a simple standing wedge, shown assembled at top.

particular, $\theta=45^{\circ}$ results in plates orthogonal to each other. This type of plate configuration is used in the closed box shown in Fig. 11.

Alternatively, with $\theta$ fixed at $45^{\circ}$, reducing $\phi$ will cause the assembled plates to be angled towards each other. An example of this arrangement is found in the steeple of the Campanile shown in Fig. 12.

\section{EXPERIMENTAL}

Single-step-assembled pop-up structures were designed and fabricated in the Sandia National Laboratories SUMMiT process [3]. Besides three structural layers of polysilicon, SUMMiT also provides $1-\mu \mathrm{m}$ design rules. 


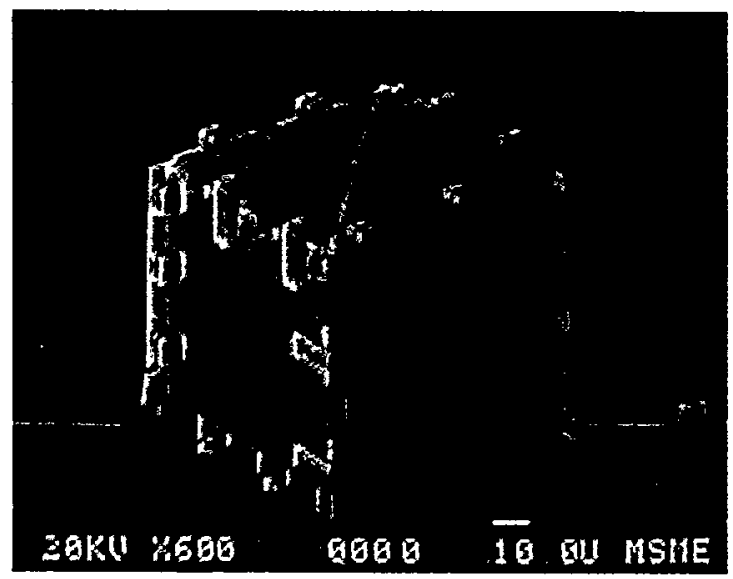

Figure 11: Pop-up box, closed on all four sides and on top. This is an example of orthogonal plates: $\phi=90^{\circ}, 2 \theta=90^{\circ}$.

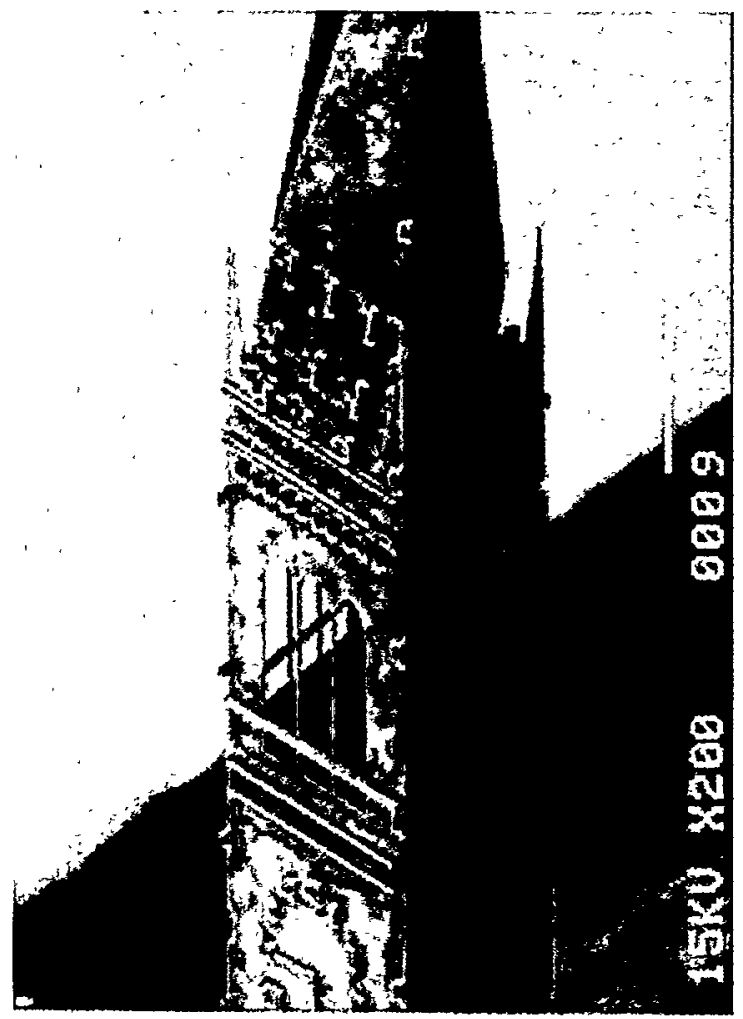

Figure 12: 3-D model of the UC Berkeley Campanile clock tower. The tower stands $1.8 \mathrm{~mm}$ high. The steeple is an example of non-orthogonal plates.

Structures were released in a 50-50 mix of HF and $\mathrm{HCl}$ for a duration of 6.5 hours. Following release, the chips were not dried, but instead, the structures remained in isopropanol for assembly. Following assembly, the structures were allowed to dry in air.

Manual assembly was accomplished by viewing through an optical microscope and manipulating structures with a MEMS Precision Instruments silicon microtweezer [4] mounted on a Sutter Instruments MP-285 motorized micromanipulator. The microtweezer was not utilized in its gripping mode. Instead, it was desirable simply as a silicon stick with the proper compliance to minimize the breaking of structures during assembly.

Assembly of these structures was successfully accomplished using a single flipping step. The recorded assembly process for a CCR is shown in Figure 13. Assembly was rapid, typically being accomplished in about $5-10$ minutes. Assembly speed is expected to be even greater with a seasoned operator and better design.

The simple assembly process would be expected translate into greater yield since there is reduced opportunity for human error (typically involving smashing or snapping a delicate polysilicon plate). However, yield was still low due to the tendency for hinges to jam, as described above. The alternative substrate hinges were observed to assemble much more reliably. However, being an experimental design, they were sparingly used, and even where used, they could not be applied for every single hinge in a structure, only being available at the lower levels of polysilicon.

Parallel self-assembly was observed as some structures were unintentionally assembled during the post-release rinse in water (Fig. 14). It should be noted that design for single-step assembly increases the complexity of structures that can be assembled by simple fluid agitation.

\section{CONCLUSION}

Pop-up design enables assembly of complex three-dimensional microstructures that is fast, 

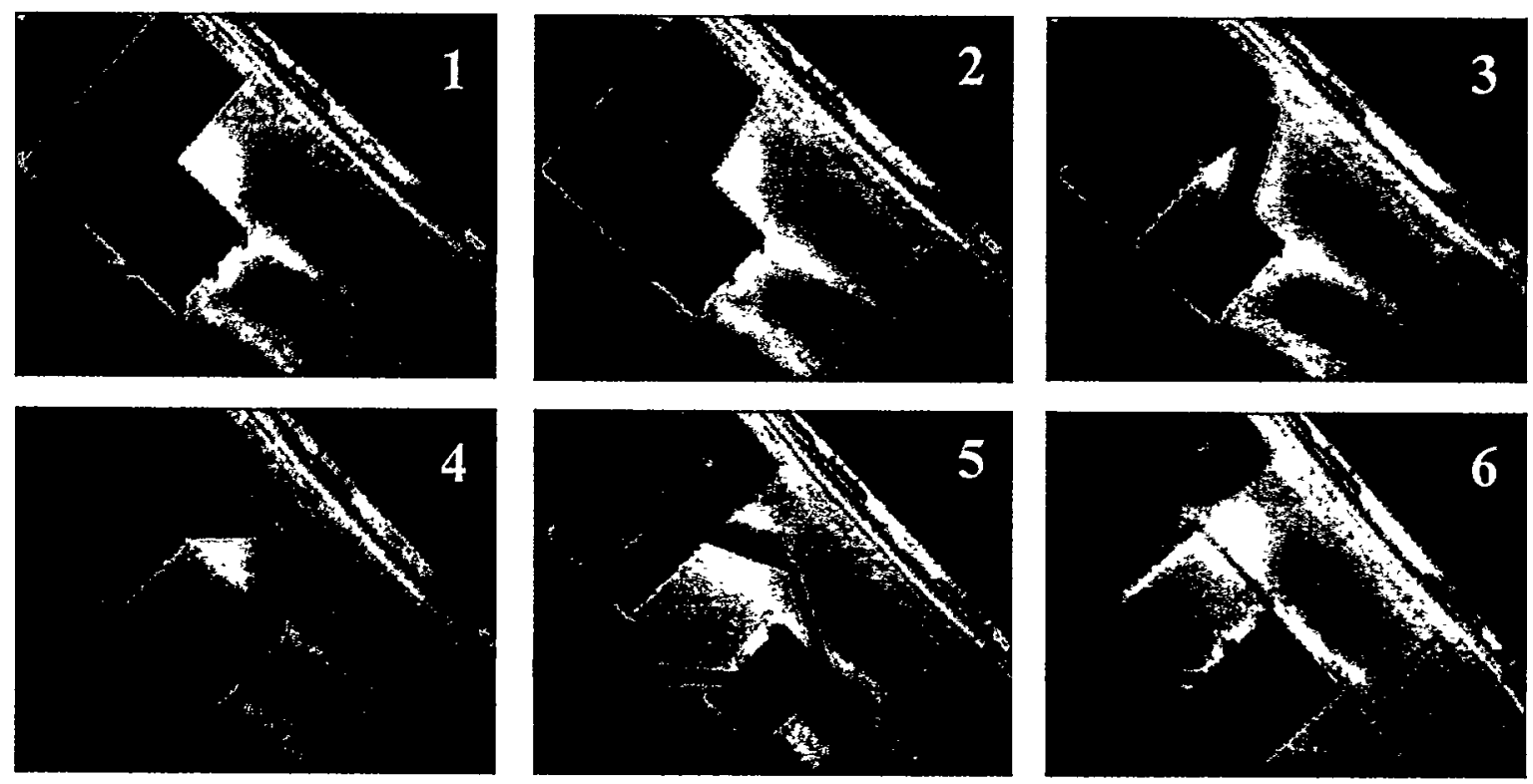

Figure 13: Optical microscope snapshots of pop-up corner-cube-reflector as it is being assembled

and, with greater attention to hinge design, potentially high yield. These manufacturing improvements may be a significant step towards the volume production of three-dimensional MEMS products.

State-of-the-art MEMS processes such as SUMMiT V [6], offering four structural layers, offer the possibility for even more intricate pop-up designs, including structures optimized for parallel self-assembly.

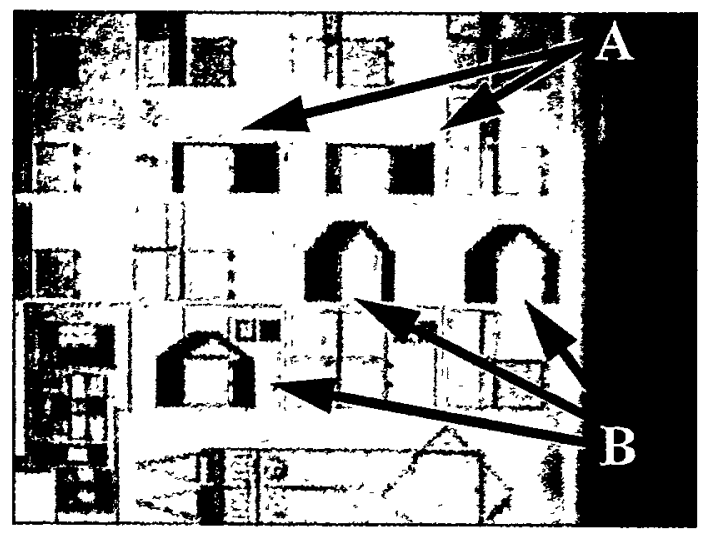

Figure 14: Due to simply the fluidic agitation of the post-release rinse, two CCRs have been assembled (A), and three more have been partially assembled (B).

\section{ACKNOWLEDGMENTS}

This material is based upon work supported under a National Science Foundation Graduate Fellowship.

Sandia is a multiprogram laboratory operated by Sandia Corporation, a Lockheed Martin Company, for the United States Department of Energy under Contract DE-AC04-94AL85000.

\section{REFERENCES}

[1] K. J. S. Pister, et. al., Sensors and Actuators A, 33 (1992) 249-256.

[2] P. B. Chu, et. al., Proc. MEMS-97, pp. 3505, Nagoya, Japan.

[3] J. J. Sniegowski, Proc. of the ASME Aerospace Division, AD-Vol. 52, pp.751-759.

[4] For more information, please refer to: http://www.memspi.com

[5] M. B. Cohn, et. al., Proc. of the SPIE, vol.3511, 1998. p.2-16.

[6] M. S. Rodgers, et. al., Proc. Transducers '99, pp. 990-3, Sendai, Japan. 